\title{
A Cost Model for Hybrid Storage Systems in a Cloud Federations
}

\author{
Amina Chikhaoui, Kamel Boukhalfa \\ University of Science and Technology Houari \\ Boumediene, Algiers, Algeria \\ \{achikhaoui, k.boukhalfa\} dusthb.dz
}

\author{
Jalil Boukhobza \\ Univ. Bretagne Occidentale \\ UMR 6285, Lab-STICC F-29200, Brest, France \\ boukhobza@univ-brest.fr
}

\begin{abstract}
A cloud federation gives to cloud service providers (CSP) the opportunity to collaborate in order to offer a better QoS to customers at a lower cost. To do so, CSPs make some spare resources available to others at a reduced cost. One of the most critical resources is the storage system as it represents the main system bottleneck. From this point of view, how to efficiently place data in a federation of Clouds with heterogeneous storage systems is a real challenge. To address this issue, one needs to accurately estimate the data placement cost. In this paper, we propose a cost model for hybrid storage systems in a cloud federation for a Database as a Service (DBaaS) application. It takes into account the storage system characteristics, customers I/O workloads and SLA. The proposed cost model considers both 1) Internal customers data placement cost including local placement, outsourcing, back-migration and penalty costs, and 2) External customers data placement cost including insourcing and geo-migration costs. It can be used to help in the decision-making process which aims to enhance customers QoS and reduce CSPs costs in a federation. Simulation results showed the relevance of the considered costs. We have shown that mis-considering some sub-costs may lead to a $95 \%$ cost error for external customers data placement and $80 \%$ for outsourcing customers. This may cause significant financial loss.
\end{abstract}

Index Terms-Cloud, federation, hybrid storage, cost model.

\section{INTRODUCTION}

C LOUD federation [1], [2], [3], [4] is a computing paradigm that consists in making several Cloud Service Providers (CSPs) cooperate by sharing resources. These CSPs insource and outsource their customers' data and services to provide continuous provisioning by exploiting temporal and spatial availability of resources [2] while reducing their cost. Cloud federation need was driven by novel applications such as mobile cloud, IoT, and big data. It came to address many limitations such as resource contention [5], [6], service interruption [7] and Quality of Service (QoS) degradation that may be due to the geographical distance to cloud resources.

DataBase as a Service (DBaaS) is one of the most important application processing Cloud service offered to cut the IT costs. For such a service, I/O performance and network latency are the two main metrics considered by the customers. Indeed, I/O system is one of the main system bottlenecks [8]. Moreover, it takes about $90 \%$ of the transaction execution time in some database queries [9].

Some cloud companies [10] already include latency guarantees in their Service Level Agreements (SLAs) and customers may pay extra charges for reducing I/O latencies.
In order to handle $\mathrm{I} / \mathrm{O}$ bottlenecks, CSPs rely on the heterogeneity of storage devices. While Hard Disk Drives (HDDs) are used mainly to provide large storage volumes thanks to their low cost per GB, flash based Solid State Drives (SSDs) are integrated to reduce access latency and increase the I/O bandwidth [27], [28]. However, their higher cost does not allow for a massive use [29]. So, according to customers' QoS requirements, a CSP may use this heterogeneity to migrate or replicate their data between different storage classes or to other partner CSPs in the case of a Cloud federation. Dealing with such local and external storage system heterogeneity makes data placement strategies very challenging and needs an accurate cost estimation in order to make adequate decisions when placing data objects.

The cost of operating a Cloud is too significant to be ignored [30], as a matter of fact, using an accurate cost model is a critical issue. In effect, cost models are frequently used for optimization sake. Several state-of-the-art studies dealt with cost estimation of storage systems. We classified them in two categories. The first category is related to studies on centralized Clouds such as [12], [13], [16], [15], [11], [31] They investigated several issues related to I/O efficiency in case of heterogeneous storage systems, see Table Ia. The second category is related to the cost estimation in the case of interconnected Clouds. These cost models dealt with different operations such as: initial placement of VMs [6], [17], [25], storage cost and geo-migration [21], [18], [32]. However, none of these studies took into account hybrid storage systems and I/O related cost, see Table Ib. They mainly used a fixed storage cost related to data volume without considering I/O operations cost (IOPS or latency).

In the case of a Cloud federation, for an accurate storage cost estimation, CSPs must consider both local and external storage costs. Indeed, it might be more cost effective to store a customer's data on a distant HDD than on a local SSD in case of customer mobility for instance. To consider this issue, the cost estimation must take into account both the details of the storage system and the properties of the Cloud federation. To the best of our knowledge these issues were not considered simultaneously in the existing work.

In this paper, we propose a model to evaluate the storage cost of object placement for DBaaS applications in a federated Cloud. We define an object as any logical entity of a database such as a table, a view, or an index. Our model consists of: 
TABLE I: Related work classification

\begin{tabular}{|c|c|c|c|c|c|}
\hline work & \multicolumn{5}{|c|}{ Hybrid storage system based cost models } \\
& Occupation & Energy & Wearout & Migration & Penalty \\
\hline$[11]$ & & & $\mathrm{x}$ & & \\
\hline$[12]$ & & & $\mathrm{x}$ & & \\
\hline$[13],[14]$ & $\mathrm{x}$ & $\mathrm{x}$ & & & \\
\hline$[15]$ & & $\mathrm{x}$ & $\mathrm{x}$ & $\mathrm{x}$ & $\mathrm{x}$ \\
\hline$[16]$ & $\mathrm{x}$ & $\mathrm{x}$ & $\mathrm{x}$ & $\mathrm{x}$ & $\mathrm{x}$ \\
\hline \multicolumn{7}{|c}{ (a) } \\
\hline
\end{tabular}

- Internal customers object placement cost: It takes into account (1) the cost of object placement for internal customers in the local infrastructure, (2) the cost of outsourcing local customers objects, that is buying resources from the other federation CSPs, (3) the cost of prospective back-migration that is the cost induced by bringing back previously outsourced internal customers objects and finally (4) the penalty cost related to the violation of customers SLAs.

- External customers placement cost: this is the cost of managing external customer objects. It consists of (1) the cost of insourcing external customers objects and (2) the cost of sending back these objects to their home or other clouds of the federation when requested.

We have evaluated the cost model and showed that the considered sub-costs are all relevant. With a simple use case, the lowest considered sub-cost part was $6 \%$. Our cost model makes it possible to investigate whether outsourcing is relevant in a federation according to the resource cost and workload properties. It also provides the CSP with a mean to evaluate the penalty cost related to outsourcing some objects and to tune the resources to provide for the federation.

The paper is organized as follows. In Section 2, we discuss some related work, then, we define the system model and formulate the problem in Section 3. In Section 4, our cost model is presented and it is evaluated in Section 5. Finally, we conclude and give some perspectives in Section 6.

\section{RELATED WORK}

In this section we discuss existing studies about hybrid storage systems and interconnected clouds cost models.

\section{A. Hybrid storage system cost models for the Cloud}

Many efforts have been made to estimate the cost of data placement in hybrid storage systems. Existing work focused mainly on single clouds. In [13], [14] the storage system cost was estimated using occupation and energy costs. Authors of [11] considered only the wear out while migration cost has been modeled in [12]. Recently, in [15], a cost model has been proposed taking into account four factors: energy, wear out limit, migration between storage classes and penalties. The authors in [16] have extended the previous study with the storage occupation cost. This is summarized in Table Ia.

\section{B. Interconnected cloud cost models}

Many existing studies [18], [19], [20] have dealt with the cost of running big data applications, such as social networks,

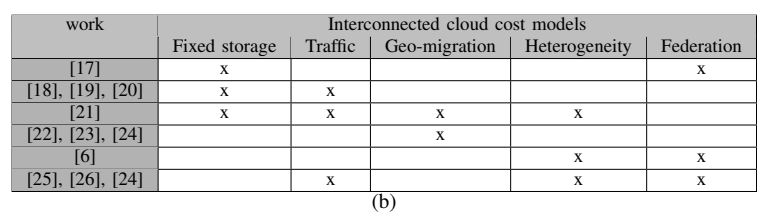

and scientific computing [33]. These studies consider storage, computation and bandwidth to evaluate the cost of data geomigration and storage in distributed data-centers. All these cost models have used a fixed storage cost mainly related to the storage occupation. The running I/O workload has not been considered. Other studies investigated the cost of distributed clouds (eg: Amazon, Google, etc.). Cost models in this category addressed different aspects: Data center construction [34], energy cost [22], [23], [24], and bandwidth cost [24]. Finally, various cost models for federated clouds have been proposed. Toosi et al [6] designed a cost model which sets dynamically the cost of federated VMs according to the amount of idle resources of the CSPs, as well as a revenue model to maximize the CSP profit. In [17] a cost model, including insourcing and outsourcing costs of virtual resources was proposed. In [25], [26], the cost model consists in local, insourcing, outsourcing and network traffic costs.

Existing hybrid storage cost models are not applicable when it comes to interconnected clouds because data placement in this case implies other factors related to the distributed environment. Regarding federated clouds, the cost of $\mathrm{I} / \mathrm{O}$ system has not been considered in detail. Indeed this cost has been generally considered as constant and was not related to application workloads.

In this paper, we propose a cost model for the storage system in a federated Cloud taking into account in one hand, the detailed cost of the storage system and in the other hand, the distributed nature and federation properties.

\section{SySTEM MODEL AND PROBLEM DEFINITION}

In this section, we first describe the system model and then we formulate the cost modeling problem.

\section{A. System model}

We consider a federation $\mathrm{F}$ composed of $\mathrm{D}$ CSPs $\mathrm{F}=$ $\left\{C P^{d}, d \in[1 . . D]\right\}$ cooperating in a peer-to-peer intercloud fashion as depicted in Figure 1. This architecture is inspired from [35] and was used in several studies [6], [25], [17]. The figure emphasizes on the $d$ cloud provider view.

Each $C P^{d}$ has one datacenter and accommodates different types of storage classes $S C=\left\{s c_{j}, j \in[1 . . J]\right\}$. We limit our study to two storage classes in this work: SSDs and HDDs. Each $s c_{j}$ has a limited capacity $c_{s c_{j}}$, a maximum IOPS per operation $i o p s_{o p, s c_{j}}$, a wear out $w o_{s c_{j}}$, and a purchase cost $p_{s c_{j}}$. We assume that a CSP buys a bandwidth $b w$ from an Internet provider with a purchase cost $p_{b w}$. 


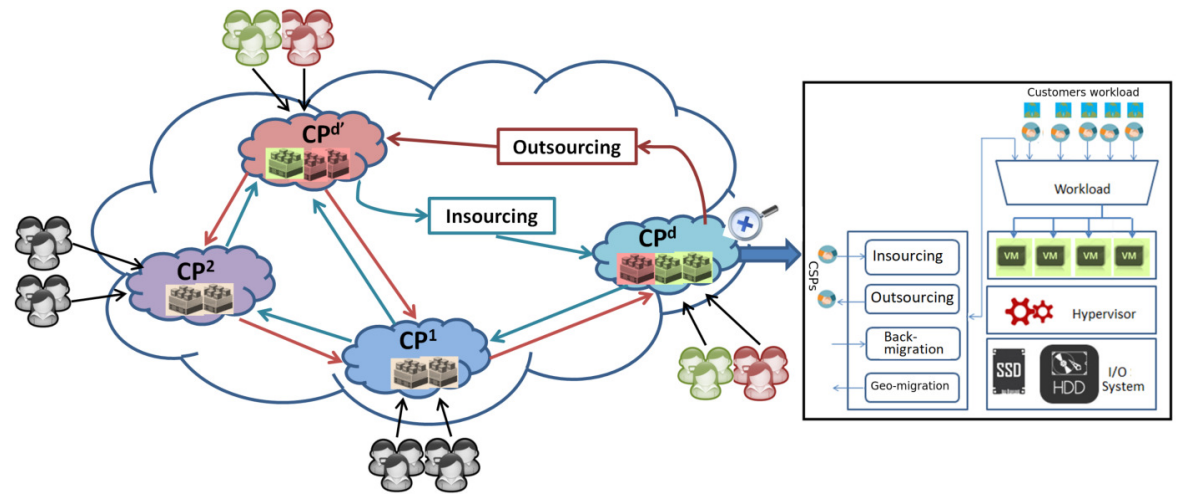

Fig. 1: Federated cloud based hybrid storage system

CSPs may outsource their customers objects to other federation members (called partners), for instance, when they cannot meet customers QoS requirements.

Therefore, each $C P^{d}$ has two types of customers:

1) internal customers: that are the customers of $C P^{d}, U_{\text {int }}=\left\{u_{k}, k \in[1, K]\right\}$,

2) external customers: that are partner CSPs $\left\{C P^{d^{\prime}}\right\}$ customers whose objects are outsourced to $C P^{d}, U_{\text {ext }}=$ $\left\{u_{k^{\prime}}^{d^{\prime}}, k^{\prime} \in\left[1, K^{\prime}\right]\right\}, C P^{d^{\prime}} \in F-\left\{C P^{d}\right\}$. These customers' objects are insourced by $C P^{d}$.

We assume that each internal customer $u_{k}$ :

1) has a set of objects $\left\{o_{i, k}, i \in[1 . . N]\right\}$. Each object has a size denoted $s_{O_{i, k}}$.

2) generates an $I / O$ workload $w l_{k}$ representing the $I / O$ operations generated by the set of queries issued by the customer. According to the access pattern (sequential, random) and the operation type (read, write), We distinguish four I/O patterns as in [16]: sequential read $(s r)$, sequential write $(s w)$, random read $(r r)$ and finally, random write $(r w)$.

3) a requested QoS (SLA) in terms of IOPS and latency iops $_{\text {sla }, k}, l t c_{\text {sla }, k}$.

4) A penalty function $p n_{k}$ composed of two parts, one related to storage performance : $p n_{i o p s, k}$ and the other to the latency: $p n_{l t c, k}$.

The same notation is used for the external customers.

Based on customers' SLA requirements and the amount of available storage resources, objects are served locally (placed and migrated between different storage classes), insourced, geo-migrated or geo-replicated to partner clouds. To clarify the system model, we introduce the following definitions:

Outsourcing [6], [36], [25], [3]: the ability that CSPs have to send some internal customers objects to other federation members. Outsourcing maybe achieved through either geomigration or geo-replication.

Geo-migration [21], [18]: the process of moving objects to other clouds without caring about local copy synchronization.

Geo-replication [21], [37]: the process of maintaining multiple copies of objects on multiple sites (CSPs) for a better performance, availability, and reliability.
Insourcing [25], [26], [36], [3]: the opposite process of outsourcing. CSPs make available part of their unused resources to respond to requests from other members.

Inner migration [16], [15]: is the movement of some objects between different storage classes within the same infrastructure. A CSP $C P^{d}$ migrates periodically some objects between the different storage classes. $O_{m, i n t}, O_{m, e x t}$ : represent the set of internal (external, respectively) customers objects to migrate between internal storage classes.

Back migration: This operation consists in bringing back previously outsourced objects to the local infrastructure.

The primary objective of a CSP is to reduce the used resources cost while meeting customers QoS needs. The cloud administrator has to take decisions about: (1) which objects need to be moved locally between different storage classes, (2) which ones need to be outsourced and to which partner CSP, i.e. which ones to be replicated or migrated, (3) which other CSP customer objects need to be insourced, (4) and finally, which previously outsourced internal customers objects, need to be brought back to the local infrastructure.

Periodically, the cloud administrator makes decisions about object placement. We note by $T$ the time period during which monitoring is executed to extract objects I/O patterns and to compute the cost of outsourced objects in order to evaluate the overall placement cost as in [16], [15]. We assume that each cloud maintains two matrices, one for internal customers objects $(A)$ and the other for external customers objects $(B)$ such as $A[i, j](T)=1$ when an internal object is placed in the cloud $C P^{j}$ and $B[i, j](T)=1$ when the object is an insourced object to cloud $C P^{j}$.

In our study, we assume that a given CSP charges its partners for insourcing actions. Reduced prices are used in order to foster cooperation within the federation. As in [6], each $C P^{d}$ dynamically adjusts the price of its contributed storage resources according to the amount of idle resources. Let $C a p_{\max _{r s c}}$ and $C a p_{i d l_{r s c}}$ be the total and idle capacities for a given resource $r s c$ of the provider $C P^{d}$. rsc maybe the storage occupation (occ) or performance in IOPS (iops). If $p_{r s c}$ is the price paid by internal customers for the resource $r s c$, its insourcing price $F_{r e s}^{d}$ is obtained from the expression 
in eq. (1) from [6]. For each time period T, CSPs will use eq. 1 to adjust their insourcing prices.

$$
F_{r s c}^{d}=\frac{\operatorname{Cap}_{\max _{r s c}}-C_{a p_{i d l_{r s c}}}}{\operatorname{Cap}_{\text {max }_{r s c}}} *\left(p_{r s c}\right)
$$

From the network resource point of view, only outgoing network (Internet) traffic is charged for customers in a given CSP, be it a local or an outsourced customer (in this case, the hosting CSP charges the external network traffic cost for outsourcing one).

In this paper, we assume that each $C P^{d}$ dedicates a fixed part of storage and bandwidth resources for its internal customers $\left(s c_{j, i n t}, b w_{i n t}\right)$ and another part for the external customers $s c_{j, e x t}, b w_{e x t}$ as noted in eq. 2 .

$$
\forall C P^{d},\left\{\begin{array}{l}
s c_{j}=s c_{j, i n t}+s c_{j, e x t} \\
b w=b w_{i n t}+b w_{\text {ext }}
\end{array}\right.
$$

\section{B. Problem formulation}

In the following, we define our problem by giving system inputs and outputs.

Input: A CSP of the federation that has : (1) a set of storage classes, (2) an Internet bandwidth with maximum capacity and purchase cost, (3) a storage and outgoing network bandwidth costs for partner Clouds. (4) a set of internal customers, (5) a set of external customers, (6) two sets of objects $O_{m, i n t}$ and $O_{m, e x t}$ to move between the local storage classes. (7) a monitoring period $T$, and (8) two matrices: $A(T)$ and $B(T)$.

Output: The monetary placement cost of all customers' objects for a given time period $T$.

\section{Storage Cost Model in a Cloud Federation}

\section{A. Overview}

We model the cost of object placement for a cloud $C P^{d}$, $d \in[1 . . D]$, belonging to a federation $F$ for a given period of time $T$. The model is built hierarchically, see Figure 2.

The total object placement cost $\operatorname{Cost}_{p l c, T}$ is the sum of the placement cost of the internal customers $\left(\operatorname{Cost}_{p l c_{i n t}, T}\right)$ and the external customers $\left(\operatorname{Cost}_{p l c_{e x t}, T}\right)$ as shown in eq. 3 .

Note that non-recurring costs which do not depend on the objects placement like maintenance cost, human resources cost, air-conditioning costs are not considered in this paper. We do not also consider the cost related to data security.

$$
\operatorname{Cost}_{p l c, T}=\operatorname{Cost}_{p l c_{i n t}, T}+\operatorname{Cost}_{p l c_{e x t}, T}
$$

B. Internal customers object placement cost (see (2), Figure 2)

This is the cost of placing internal customers objects. It includes the local placement cost $\operatorname{Cost}_{l c l_{i n t}, T}$, the outsourcing cost $\left(\right.$ Cost $_{\text {out }}$ src $\left._{,}, T\right)$, the back-migration cost $\left(\right.$ Cost $\left._{b c k_{m g r}, T}\right)$ and the penalty cost $\operatorname{Cost}_{p n t_{i n t}, T}$ as shown in eq. 4 .

The local placement cost is the storage cost of internal customers objects in the local infrastructure. The outsourcing cost is related to the placement of internal customers objects in partner CSPs. The back-migration cost represents the cost of bringing back the previously outsourced objects to the home infrastructure. Finally, the penalty cost represents the additional monetary cost caused by SLA violations.

$$
\begin{aligned}
& \operatorname{Cost}_{p l c_{\text {int }}, T}=\operatorname{Cost}_{\text {lcl }_{\text {int }}, T}+\operatorname{Cost}_{\text {out }_{s r c}, T^{+}}+ \\
& \text {Cost }_{b c k_{m g r}, T}+\text { Cost }_{\text {pnt }} \text { int }, T
\end{aligned}
$$

1) Local placement cost (see (4), Figure 2): It is obtained from the storage cost $\operatorname{Cost}_{s t g_{i n t}, T}$ of internal customers objects and the inner migration cost (between storage classes within the CSP infrastructure) Cost $_{m g r_{i n t}, T}$ of the set of objects $O_{m, i n t}$ as shown in eq. 5 .

$$
\operatorname{Cost}_{l c l_{i n t}, T}=\operatorname{Cost}_{\text {stg }_{\text {int }, T}}+\text { Cost }_{m g r_{i n t}, T}
$$

The storage cost: As in [16], Cost $_{s t g_{i n t}, T}$ is related to the occupation Cost $_{o c c, T}$, the energy Cost $_{\text {erg }, T}$ and the wear out cost Cost $_{e d r, T}$ due to I/O workload (see eq. 6).

$$
\operatorname{Cost}_{\text {stg }_{\text {int }}, T}=\max \left[\operatorname{Cost}_{\text {occ }, T}, \text { Cost }_{\text {edr }, T}\right]+\text { Cost }_{\text {erg }, T}
$$

The occupation cost is the amortized cost of the storage system over the period $T$. The energy cost is the energy consumed by the storage system to execute the I/O workload multiplied by the energy unitary price which we consider constant. Finally, the wear out cost is caused by the execution of the I/O workload which impacts the lifetime of the devices. For SSD, this cost is related to the amount of written data while for HDD, it depends of the amount of both read and written data. For more details see [16].

The migration cost: it consists in reading the set of objects $O_{m_{\text {int }}}$ from the source storage device and writing them to another destination storage device. It includes the energy and endurance costs.

2) Outsourcing cost (see (5), Figure 2): It is composed of geo-migrating and geo-replicating internal customers objects and the storage cost in the partner CSPs generated by these two geo-operations as in eq. 7.

$$
\text { Cost }_{\text {out }_{s r c}, T}=\text { Cost }_{\text {geo }_{m g r}, T}+\text { Cost }_{\text {geo }_{r p l}, T}+\text { Cost }_{\text {ext }}{ }_{p l c}, T
$$

The geo-migration cost (see (11), Figure 2): geo-migration of objects incurs local read operations $\operatorname{Cost}_{r d, T}$ and internet bandwidth usage $\operatorname{Cost}_{b w, T}$.

The set of objects concerned by the geo-migration $O_{g_{m g r}}$ is obtained from the difference between the matrix $A$ at the period $T-1$ and the current period $T$ where $o_{i}, A[i, d](T-$ $1)=1 \wedge A[i, d](T)=0$. Geo-migration cost is shown in eq. 8 .

$$
\text { Cost }_{\text {geo }_{m g r}, T}=\text { Cost }_{r d, T}+\text { Cost }_{b w, T}
$$

The local read operation cost incurs energy $\operatorname{Cost}_{r d_{e r g}, T}$ and wear out $\operatorname{Cost}_{r d_{e d r}, T}$ costs.

$$
\operatorname{Cost}_{r d, T}=\text { Cost }_{r d_{e r g}, T}+\text { Cost }_{r d_{e d r}, T}
$$

The Internet bandwidth cost is related to the bandwidth consumed by the geo-migration of objects to external clouds. 


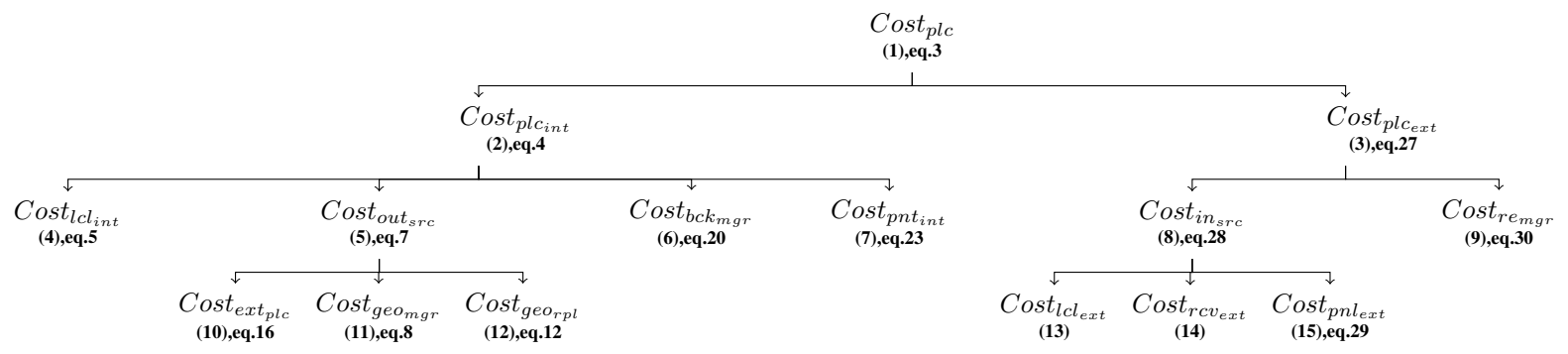

Fig. 2: The overall cost model

We calculate this cost by multiplying the size of all objects to be migrated by the amortized bandwidth over one unit of time $\operatorname{Cost}_{b w_{a m z}, 1}\left(b w_{i n t}\right)$.

$$
\operatorname{Cost}_{b w, T}=\frac{\sum_{o_{i} \in O_{g_{m g r}}}\left(S_{o_{i}}\right) * \operatorname{Cost}_{b w_{a m z}, 1}\left(b w_{i n t}\right)}{b w_{i n t}}
$$

We calculate the amortized bandwidth cost over one unit of time by distributing the purchase cost of bwint over the subscription time period $T_{s p}$.

$$
\operatorname{Cost}_{b w_{a m z}, 1}\left(b w_{i n t}\right)=\frac{p_{b w} * \frac{b w_{i n t}}{b w}}{T_{s p}}
$$

The geo-replication cost (see (12), Figure 2): georeplicating objects over multiple CSPs consumes supplementary storage and network costs. The geo-replication consists in adding, deleting and synchronizing replicas. So, its overall cost is the sum of the aforesaid operations costs as shown in eq. 12. Deleting replica cost is assumed to be nil.

$$
\operatorname{Cost}_{\text {geo }_{r p l}, T}=\operatorname{Cost}_{a d d_{r p l}, T}+\operatorname{Cost}_{s y c_{r p l}, T}
$$

The cost of adding replicas is the same as the geo-migration cost without deleting the original copy and considering the set of objects $\left\{O_{g_{r p l}}^{d^{\prime}}, d^{\prime} \neq d\right\}$. They are obtained from matrix $A$.

$$
\operatorname{Cost}_{a d d_{r e p}, T}=\sum_{C P^{d^{\prime}} \in F-\left\{C P^{d}\right\}} \operatorname{Cost}_{g_{e o_{m g r}, T}}\left(O_{g_{r p l}^{d^{\prime}}}^{d^{\prime}}\right)
$$

Concerning replica synchronization either $C P^{d}$ forwards the synchronization or receives it. $O_{s_{r p l}}^{d^{\prime}}$ is the list of objects sent to, or received from $C P^{d^{\prime}}$ for synchronizing replicas. When $C P^{d}$ forwards the synchronization, its cost is the sum of the read operations induced and the consumed Internet bandwidth costs, see eq. 14 .

$$
\operatorname{Cost}_{\text {syn }_{r e p}, T}=\sum_{C P^{d^{\prime}} \in F-\left\{C P^{d}\right\}} \operatorname{Cost}_{\text {geo } m g r, T}\left(O_{s_{r p l}}^{d^{\prime}}\right)
$$

Whereas when $C P^{d}$ receives the synchronization, this cost consists of writing operations, local Internet bandwidth use, and outgoing network costs (i.e back-migration cost, see later in eq. 20) as shown in the next equation 15.

$$
\text { Cost }_{\text {syn }_{r e p}, T}=\sum_{C P^{d^{\prime}} \in F-\left\{C P^{d}\right\}} \operatorname{Cost}_{b c k_{m g r}, T}\left(O_{s_{r p l}}^{d^{\prime}}\right)
$$

The external placement cost (see (10), Figure 2): Once the objects have been migrated to other clouds, the CSP needs to pay partner clouds for hosting the objects. Therefore, the external storage cost includes the external occupation cost Cost $_{\text {ext }}$ occ $_{T}, T$, the external workload execution cost Cost $_{\text {ext }} w_{\text {wld }}, T$ and the external penalty cost $\operatorname{Cost}_{\text {ext }}$ pnl,$T$. The penalty cost here is the charge paid by the partner clouds in case SLAs are violated for the outsourced objects.

The purchased resources are billed according to eq. 1 .

$$
\operatorname{Cost}_{e x t_{p l c}, T}=\operatorname{Cost}_{e x t_{o c c}, T}+\text { Cost }_{e x t_{w l d}, T}-\operatorname{Cost}_{e x t_{p n l}, T}
$$

The external occupation cost: represents the cost of storing the migrated or replicated internal customers objects on partner clouds. We calculate this cost by using the federated occupation cost $F_{o c c}^{d^{\prime}}$ of $C P^{d^{\prime}}$ as noted in eq. 17

$$
\text { Cost }_{\text {ext }}{ }_{o c c}, T=\sum_{d^{\prime} \mid C P^{d^{\prime}} \in F-\left\{C P^{d}\right\}}\left(\sum_{i} A\left[i, d^{\prime}\right] * S\left(o_{i}\right)\right) * F_{o c c}^{d^{\prime}}
$$

The cost of workload execution represents the amount of expected IOPS consumed by internal users. It is calculated the same way as in the eq. 18 where $F_{\text {iops }}^{d^{\prime}}$ is the cost of one IOPS in the cloud $C P^{d^{\prime}}$

$$
\begin{aligned}
& \text { Cost }_{\text {ext }}{ }_{w l d}, T \\
& =\sum_{d^{\prime} \mid C P^{d^{\prime}} \in F-\left\{C P^{d}\right\}}\left(\sum_{i} A\left[i, d^{\prime}\right] * \operatorname{IOP} S\left(o_{i}\right)\right) * F_{i o p s}^{d^{\prime}}
\end{aligned}
$$

The penalty cost is a percentage $\alpha \%$ of the total charges paid by $C^{p}$ to the partner cloud, as in Amazon Cloud.

$$
\text { Cost }_{\text {ext }}^{p n l, T}=\alpha *\left(\text { Cost }_{e x t_{o c c}, T}+\text { Cost }_{\text {ext }_{w l d}, T}\right)
$$

3) Back migration cost (see (6), Figure 2): Cost $_{b c k_{m g r}, T}$ is the cost of bringing back the previously outsourced objects to the local infrastructure. It is composed of the cost of data transfer (outgoing network cost) from the partner clouds $\operatorname{Cost}_{n t w_{\text {out }}, T}$, the local Internet bandwidth cost $\operatorname{Cost}_{b w, T}$ 
corresponding to the bandwidth consumed locally to receive the previously outsourced objects, and the cost $\operatorname{Cost}_{w r, T}$ to write the received objects on the local storage, see eq. (20).

$$
\operatorname{Cost}_{b c k_{m g r}, T}=\operatorname{Cost}_{w r, T}+\operatorname{Cost}_{n t w_{o u t}, T}+\operatorname{Cost}_{b w, T}
$$

The set of internal customers objects to be brought back $O_{b_{m g r}}$ is deduced from the matrix $A$.

The local write cost: is the cost of placing previously outsourced objects in the local storage system. This cost involves the cost of energy $\operatorname{Cost}_{w r_{e r g}, T}$ consumed by the storage devices and the wear out cost of these devices Cost $_{w r_{e d r}, T}$ caused by the write operations. Some of these objects are placed on HDD and others on SSD, see eq. 21.

$$
\text { Cost }_{w r, T}=\text { Cost }_{w r_{e r g}, T}+\text { Cost }_{w r_{e d r}, T}
$$

Outgoing network cost: This cost is charged by partner CSPs when $C P^{d}$ brings back their internal customer objects to the local infrastructure. We calculate this cost by multiplying the size of the objects to bring back by the cost of outgoing network Cost $_{\text {out }}\left(C P^{d^{\prime}}\right)$ of the partner.

$$
\begin{aligned}
\text { Cost }_{n t w_{\text {out }}, T}=\sum_{C P^{d^{\prime}} \in F-\left\{C P^{d}\right\}}\left(\text { Cost }_{\text {out }}\left(C P^{d^{\prime}}\right) *\right. \\
\left.\left(\sum_{o_{i} \in O_{b_{m} g r}} S_{o_{i}}\right)\right)
\end{aligned}
$$

Local Internet bandwidth cost is calculated with eq. 10 .

4) Penalty cost (see (7), Figure 2): The violation of SLA terms by the CSP entails a penalty that should be paid. As in [16], we calculate the overall penalty as the sum of all internal customers penalties.

$$
\text { Cost }_{p n t_{i n t}, T}=\sum_{u_{k} \in U_{i n t}}\left(\operatorname{Cost}_{p n t, T}\left(u_{k}\right)\right)
$$

Internal customer penalty cost is composed of a penalty

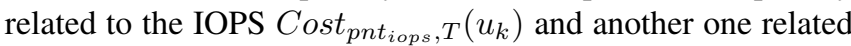
to the latency $\operatorname{Cost}_{p n t_{l t c}, T}\left(u_{k}\right)$.

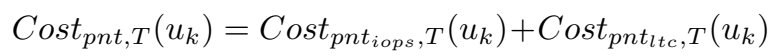

The IOPS penalty cost is calculated as in [16], it is proportional to the ratio between the offered IOPS iops $s_{\text {of fered }}\left(u_{k}\right)$ and the requested one $\left(\right.$ iops $\left._{s l a, k}\right)$. The requested IOPS is defined in the SLA while we calculate the offered IOPS from the time needed to handle the $\mathrm{I} / \mathrm{O}$ workload of the customer $u_{k}$ and the total number of $\mathrm{I} / \mathrm{O}$ requests issued to their objects. It is calculated as follows:

$$
\operatorname{Cost}_{\text {pnt }_{\text {iops }, T}}\left(u_{k}\right)=\text { pnt }_{\text {iops }, k}\left(\frac{\text { iops }_{\text {offered }}\left(u_{k}\right)}{\text { iops }_{\text {sla }, k}}\right)
$$

The latency penalty cost: In our model, we assume that customers pay extra charges to have a reduced network latency. If the latency offered $l t c_{\text {offered }}\left(u_{k}\right)$ to customer $u_{k}$ is lower than the one requested $l t c_{s l a, k}$, then a penalty is applied to the CSP. This penalty cost is proportional to the violation degree and the number of requests $n b_{r q t}$, as noted in Table VI. We calculate the latency penalty cost as follows:

$$
\operatorname{Cost}_{l t c_{i o p s}, T}\left(u_{k}\right)=\sum_{i=1} n b_{r q t}\left(p n_{l t c, k}\left(\frac{l t c_{o f f e r e d}\left(u_{k}\right)}{l t c_{s l a, k}}\right)\right)
$$

TABLE II: Latency penalty per request

\begin{tabular}{cc}
\hline$l t c_{\text {offered }}$ & Penalty $(\%)$ \\
\hline$\left[0 . . B_{0}\right]$ & 0 \\
{$\left[B_{i} . . B_{i+1}\right]$} & $x_{i}$ \\
$>B_{n}$ & $x_{n}$ \\
\hline
\end{tabular}

\section{External customers placement cost (see (3), Figure 2)}

This cost Cost $_{p l c_{e x t}, T}$ is the sum of the cost of insourcing external customers objects $\operatorname{Cost}_{i n_{s r c}, T}$ and the cost generated when some objects of the external customers are geo-migrated to other clouds or taken back by their home clouds (remigration cost) $r e_{m g r}$, see eq. 27.

$$
\operatorname{Cost}_{p l c_{e x t}, T}=\text { Cost }_{i n_{s r c}, T}+\text { Cost }_{r e_{m g r}, T}
$$

1) Insourcing cost (see (8), Figure 2): The insourcing cost is composed of receiving external customers objects cost Cost $_{r c v_{e x t}, T}$, the local placement cost $\operatorname{Cost}_{l c l_{e x t}, T}$ and the penalty cost $\operatorname{Cost}_{p n t_{e x t}, T}$.

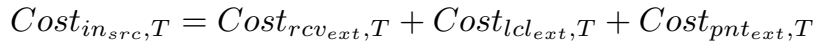

Receiving cost (see (14), Figure 2) of the external customers objects is the cost of writing these objects, calculated from eq. 21, and the cost of the consumed local Internet bandwidth, calculated with eq. 10 . The set of external customers objects to be received $O_{e_{r c v}}$ is deduced from the matrix $B$

The local placement cost (see (13), Figure 2): of the external customers objects is calculated from eq. 5 .

The penalty cost (see (15), Figure 2) is the sum of penalty costs of the external customers.

$$
\operatorname{Cost}_{p n t_{e x t}, T}=\sum_{u_{k^{\prime}}^{d^{\prime} \in U_{\text {ext }}}}\left(\operatorname{Cost}_{p n t, T}\left(u_{k^{\prime}}^{d^{\prime}}\right)\right)
$$

In the federation, resource prices are dynamically set (see eq. 1), so the penalty cost is set accordingly and is related to the unused storage resources $\beta$ which is calculated as follows:

$$
\beta=\frac{\operatorname{Cap}_{\text {max }_{r s c}}-C_{a p_{i d l_{r s c}}}}{\operatorname{Cap}_{\text {max }_{r s c}}}
$$

With $r s c$ being a combination of the storage space and performance requested. The penalty cost of an external customer $u_{k^{\prime}}^{d^{\prime}}$ is thus given in eq. 31 :

$$
\operatorname{Cost}_{p n t, T}\left(u_{k^{\prime}}^{d^{\prime}}\right)=\beta * \operatorname{Cost}_{\text {pnt }}{ }_{\text {iops }, T}\left(u_{k^{\prime}}^{d^{\prime}}\right)
$$


2) The re-migration cost (see (9), Figure 2): This cost is driven by the decision of home clouds to geo-migrate their customers back to their infrastructure or to another cloud. This incurs local read operations and Internet bandwidth costs as described in eq. 8 . The set of objects concerned by the remigration is deduced from the matrix $B$ by the difference between the period $T-1$ and the current period $T$ where for $o_{i}, B[i, d](T-1)=1 \wedge B[i, d](T)=0$.

\section{Evaluation}

This section presents an evaluation of the proposed cost model. Our aim is twofold: (i) validating the relevance of the sub-costs used in our cost model and comparing to state-ofthe-art models, (ii) showing the flexibility of the cost model through the investigation of the impact of different parameters on the placement cost.

\section{A. Experimental Settings}

We used CloudSim simulator [38]. The simulated scenario is composed of a federation of 9 geographically distributed CSPs. Each CSP is composed of one datacenter running 1000 VMs as in [6]. Some of these VMs (set to $20 \%$ in our experiments) are devoted to the federation. Each CSP has an internet bandwidth of $1 \mathrm{Gbps}$ bought from an Internet service provider (the price was set to $1500 \$$ per month). We used a standard VM configuration with 8 cores, 8 GB of RAM and a hybrid storage system. Characteristics of the storage system is provided in Table III.

TABLE III: Storage devices specifications

\begin{tabular}{|c|c|c|}
\hline Characteristics & HDD & SSD(\$) \\
\hline Price $(\$)$ & 230 & 200 \\
\hline Size & $1 \mathrm{~TB}$ & $128 \mathrm{~GB}$ \\
\hline \multirow{2}{*}{ Performance } & Seek time: $8.5 \mathrm{~ms}$ & rr:10000 IOPS, rw:40000 IOPS \\
\cline { 2 - 3 } & read/write: $9.5 \mathrm{~ms}$ & sr:540 MB/s, sw: $520 \mathrm{MB} / \mathrm{s}$ \\
\hline
\end{tabular}

Each CSP manages a set of databases (DBs) built using TPC-H and TPC-C benchmarks with different sizes and varied workload, we used the configuration given in [16]. For more details about the different DBs and storage system specifications see Table IV. The amount of storage penalty is set to $30 \%$ of the total charges paid by the customer as in Amazon Cloud. The storage price is fixed according to Amazon gp2 price model $(0.1 \$ / \mathrm{GB} /$ month $)$ while the energy cost is set to $0.1 \$$ per $\mathrm{kWh}$ as in [13].

Some of the customers are mobile and ask for a good latency by paying some extra charge. Their home CSP generally migrates their objects to the nearest CSP that meets the latency constraint otherwise the home CSP may undergo a penalty. Mobile customers are supposed to have a 1 week duration mobility. The price of latency is given in Table V [10] and its related penalty in Table VI. The evaluation is conducted over a 1 month with one-hour time period for dynamic resource price update. The insourcing prices are dynamically set by CSPs using equation (eq. 1) and changed each hour (period $T$ ). We assume that the outgoing network cost is set to $50 \%$ of amazon's which gives 0.09\$/GB.

TABLE IV: Data bases specifications

\begin{tabular}{|c|c|c|c|c|c|c|}
\hline Data base & Bench. & Reqs. nbr $(\mathrm{op} / \mathrm{h})$ & $\operatorname{rr}(\mathrm{op} / \mathrm{h})$ & rw(op/h) & $\mathrm{sr}(\mathrm{op} / \mathrm{h})$ & $\mathrm{sw}(\mathrm{op} / \mathrm{h})$ \\
\hline $\mathrm{DB} 1(32 \mathrm{~GB} / \mathrm{HDD})$ & \multirow{2}{*}{ TPC-C } & 432000 & 136800 & 46800 & 108000 & 32400 \\
\hline $\mathrm{DB} 2(60 \mathrm{~GB} / \mathrm{SSD})$ & & 28800000 & 601200 & 104400 & 5436000 & 147600 \\
\hline DB3(147GB/HDD) & \multirow{3}{*}{ TPC-H } & 331200 & 10800 & 216000 & 3600 & 18000 \\
\hline DB4(34GB/HDD) & & 355200 & 32400 & 216000 & 7200 & 10800 \\
\hline DB5(381GB/HDD) & & 15360 & 1080 & 8280 & 360 & 1080 \\
\hline
\end{tabular}

TABLE V: Latency price/req.

\begin{tabular}{cc}
\hline Latency $(\mathrm{ms})$ & Price by request $(\$)$ \\
\hline$<200$ & 0.0000001 \\
$<400$ & 0.00000005 \\
$<600$ & 0.00000002 \\
$>600$ & 0 \\
\hline
\end{tabular}

\section{B. Evaluation results}

1) Relevance of the sub-costs: The first experiment concerns the relevance of the used sub-costs. In this scenario, the network latency between each pair of cloud infrastructures is assigned randomly between ]200ms, 700ms]. First, we evaluated all different sub-costs for one CSP, then, we calculated the average placement cost of (1) insourced databases see (8), Figure 2 and (2) outsourced databases see (5), Figure 2, and compared the resulting costs with those of some state-of-theart studies [18], [19], [21], [6], [25]. These costs were chosen as they are the higher ones.

Figure $3 \mathrm{a}$ and Figure $3 \mathrm{~b}$ show the different sub-costs of our cost model (leaf nodes of Figure 2). In this evaluation, geo-replication costs were not considered (only geo-migration has been shown). We observe that all the modeled costs are relevant as each cost is high enough for at least one tested database. We observe that the local placement and penalty costs of internal customers are the highest ones. This is due to the fact that only a small part of customers are mobile (20\%) and for small periods (1 week). Also, the database size affects directly the external placement cost because the storage in this case is bought from others CSPs. The database size affects likewise all the costs including geo-migration and back-migration, while the remaining costs are affected by the storage device type and workload patterns.

Figure $4 \mathrm{a}$ and Figure $4 \mathrm{~b}$ show the cost of insourced and

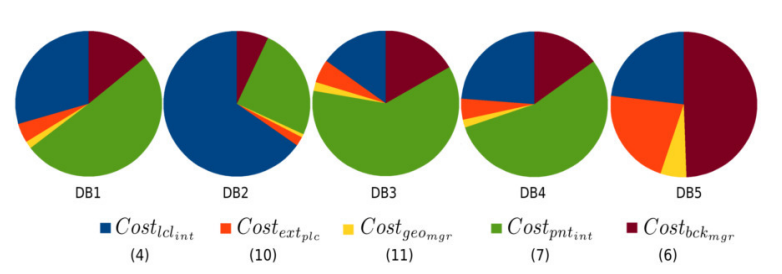

(a) Internal customers placement sub-costs

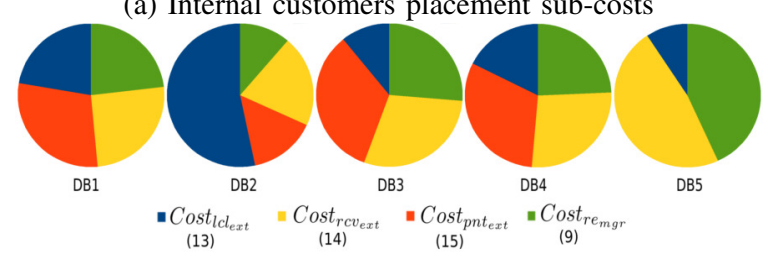

(b) External customers placement sub-costs

Fig. 3: Sub-costs of the simulated placement cost. 


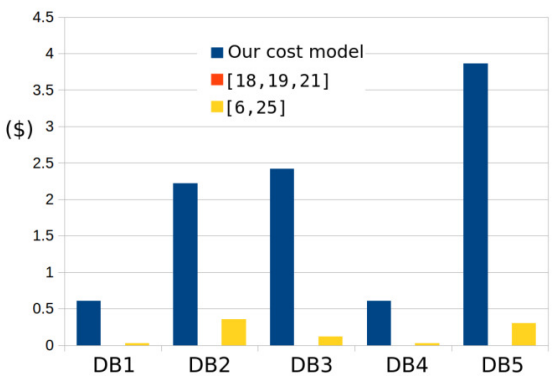

(a) Insourced database cost

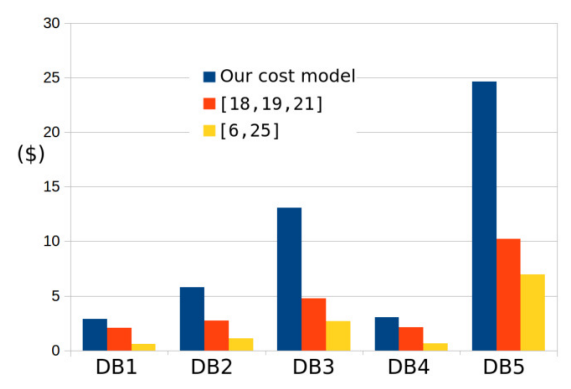

(b) Outsourced database cost

Fig. 4: The average per week placement cost of the insourced and outsourced databases for a given CSP.

outsourced databases for a given CSP for our cost model as compared to state-of-the-art models. A first observation one may draw is that models from [18], [19], [21] do not consider the insourcing cost. In addition, the cost of storage services for outsourced objects is fixed to $1 \mathrm{~GB} /$ unit of time. In [6], [25], only the occupation cost is considered with no geo-migration cost which can lead, in the worse case, to a difference with our cost model of $95 \%$ for the external customers placement cost and 80\% for the outsourcing cost. Furthermore, the penalty cost is ignored in those studies while in our work it can represent up to $61 \%$ of the placement cost of internal customers and $32 \%$ of that of external customers.

2) Spare resources pricing model evaluation: The objective of this part is to show how the proposed cost model makes it possible to easily evaluate the spare resource pricing model used according to the ran workload.

Figure 5.a shows, from one hand the cost of running insourced databases and from the other hand, the price billed to the partner clouds. As one can observe, the financial gain of insourcing objects highly depends on the nature of the ran workload. Insourcing large volumes of data generating a low number of queries (DB5) is of course more interesting.

Figure 5.b shows the outsourced objects cost when buying resources from partner clouds as compared to buying them from an external cloud. This Figure illustrates the ability of the model to highlight the gain obtained for a given CSP knowing its outsourced objects and the used federation pricing model. This may help to optimize the outsourcing decision.

3) Impact of latency and penalty, Federation vs DCC vs single Cloud: The designed cost model makes it possible to compare the cost of running a Cloud infrastructure within or out of a Cloud federation. In this part, we compare the average placement cost of mobile customers databases by varying the latency between clouds for three scenarios: (1) Geo-migration using a federation, (2) Geo-migration using a distributed cloud computing platform (DCC) and (3) Single Cloud without geomigration (see Table 6). The aim of this part is to show the impact of the violation degree and the network latency on the average placement cost for the three configurations.

We notice, from Figure 6, that generally, the cost without outsourcing mobile customers increases with the increase of the network latency. For databases with heavy workloads (e.g.
DB2) it is always interesting to outsource objects. In fact, outsourcing is interesting as long as the amount of outsourced data is small and the workload is heavy. This is not the case for data with small workload and large size. This is because geo and back-migrating these data implies a high network traffic cost (e.g. DB5). For some databases with large sizes and medium workloads, it is not cost-effective to outsource them as long as the violation of the latency is not high. However, when the degradation of network latency reaches a certain level, it becomes interesting to be outsourced. For instance, for DB3 the cost without migration (with penalty) is lower than the cost with migration when latency is $<400 \mathrm{~ms}$ but it is the opposite when the latency becomes $>600 \mathrm{~ms}$. Our cost model allows to make a trade-off between all these parameters for optimizing the overall cost of running a Cloud into a federation.

\section{CONCLUSiON}

A CSP can meet its customers QoS and minimize the cost of data placement by using either local or partner resources in a Federation. In this work, we have proposed a cost model for data placement on hybrid storage systems in a Cloud federation. Our model extends state-of-the-art work by considering geo-migration, penalty, back-migration, and georeplication costs.

For future work, we will first investigate placement optimization strategies to find the optimal internal and/or external placement for CSP objects. We will also design pricing strategies for resources in a Federation.

The performed evaluations proved the relevance of the considered costs. It also pointed out that outsourcing and insourcing is a complex task that requires taking into account a large number of parameters. Even though our model is storage oriented, it can be used and integrated in a broader cost model considering other resources (e.g. CPU and memory).

\section{REFERENCES}

[1] D. Villegas, N. Bobroff, I. Rodero, J. Delgado, Y. Liu, A. Devarakonda, L. Fong, S. M. Sadjadi, and M. Parashar, "Cloud federation in a layered service model," Journal of Computer and System Sciences, vol. 78, no. 5, pp. 1330-1344, 2012, http://dx.doi.org/10.1016/j.jcss.2011.12.017.

[2] H. Li, C. Wu, Z. Li, and F. C. Lau, "Profit-maximizing virtual machine trading in a federation of selfish clouds," in INFOCOM, 2013 Proceedings IEEE. IEEE, 2013, pp. 25-29, http://dx.doi.org/10.1109/infcom. 2013.6566728 . 

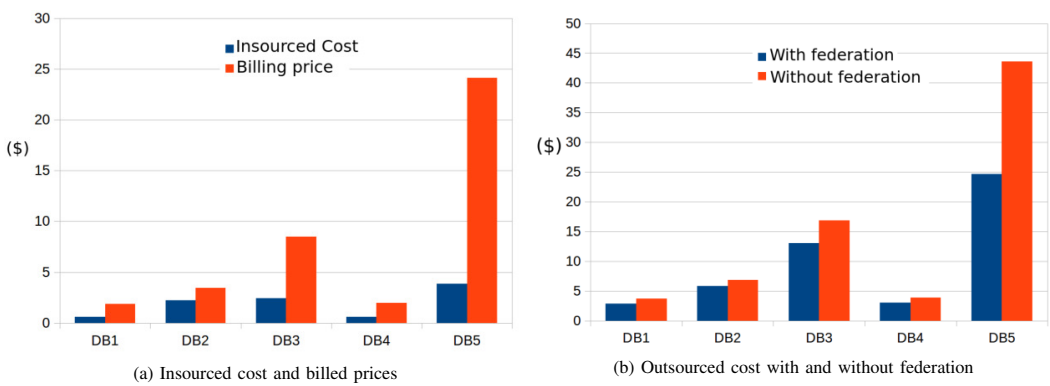

Fig. 5: Federation impact on the cost

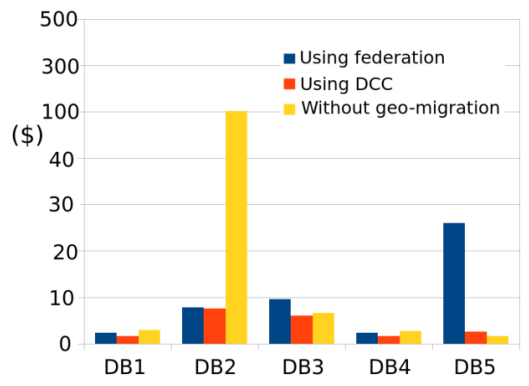

(a) $200<$ Latence $(m s)<400$

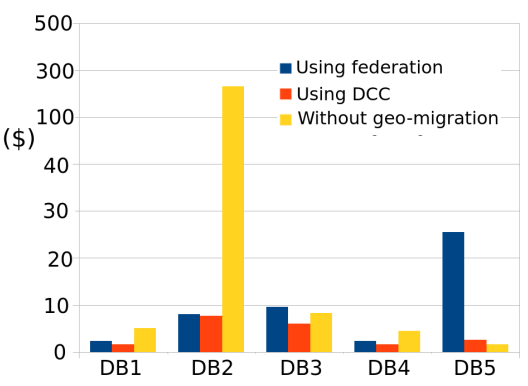

(b) $400<$ Latence $(m s)<600$

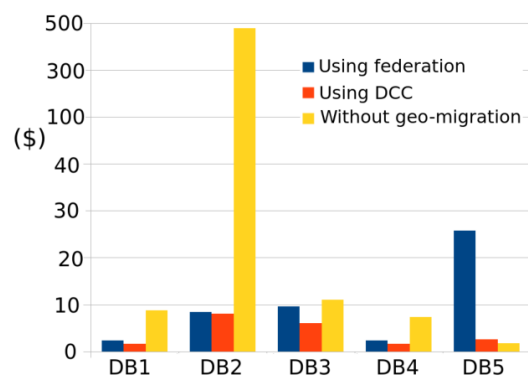

(c) Latence $(m s)>600$

Fig. 6: Average cost with migration in federated cloud and distributed cloud VS average cost without migration

[3] M. R. Assis and L. F. Bittencourt, "A survey on cloud federation architectures: identifying functional and non-functional properties," Journal of Network and Computer Applications, vol. 72, pp. 51-71, 2016, http://dx.doi.org/10.1016/j.jnca.2016.06.014.

[4] R. Moreno-Vozmediano, E. Huedo, I. M. Llorente, R. S. Montero, P. Massonet, M. Villari, G. Merlino, A. Celesti, A. Levin, L. Schour et al., "Beacon: a cloud network federation framework," in Communications in Computer and Information Science. Springer, 2016, pp. 325-337, http://dx.doi.org/10.1007/978-3-319-33313-7_25.

[5] M. Amiri and L. Mohammad-Khanli, "Survey on prediction models of applications for resources provisioning in cloud," Journal of Network and Computer Applications, vol. 82, pp. 93-113, 2017, http://dx.doi. org/10.1016/j.jnca.2017.01.016.

[6] A. N. Toosi, R. N. Calheiros, R. K. Thulasiram, and R. Buyya, "Resource provisioning policies to increase iaas provider's profit in a federated cloud environment," in High Performance Computing and Communications (HPCC), 2011 IEEE 13th International Conference on. IEEE, 2011, pp. 279-287, http://dx.doi.org/10.1109/hpcc.2011.44.

[7] Y. Gu, D. Wang, and C. Liu, "Dr-cloud: Multi-cloud based disaster recovery service," Tsinghua Science and Technology, vol. 19, no. 1, pp. 13-23, 2014, http://dx.doi.org/10.1109/tst.2014.6733204.

[8] E. Shriver, "Performance modeling for realistic storage devices," 1997, https://dl.acm.org/citation.cfm?id=269078.

[9] M. A. Sharaf, P. K. Chrysanthis, A. Labrinidis, and C. Amza, "Optimizing i/o-intensive transactions in highly interactive applications," in Proceedings of the 2009 ACM SIGMOD International Conference on Management of data. ACM, 2009, pp. 785-798, http://dx.doi.org/10. 1145/1559845.1559927.

[10] D. B. Terry, V. Prabhakaran, R. Kotla, M. Balakrishnan, M. K. Aguilera, and H. Abu-Libdeh, "Consistency-based service level agreements for cloud storage," in Proceedings of the Twenty-Fourth ACM Symposium on Operating Systems Principles. ACM, 2013, pp. 309-324, http://dx. doi.org/10.1145/2517349.2522731.

[11] Y. Cheng, M. S. Iqbal, A. Gupta, A. R. Butt, and V. Tech, "Pricing games for hybrid object stores in the cloud: Provider vs. tenant." in HotStorage, 2015, https://www.usenix.org/conference/hotcloud15/ workshop-program/presentation/cheng.

[12] L. Lin, Y. Zhu, J. Yue, Z. Cai, and B. Segee, "Hot random off-loading: A hybrid storage system with dynamic data migration," in Modeling,
Analysis \& Simulation of Computer and Telecommunication Systems (MASCOTS), 2011 IEEE 19th International Symposium on. IEEE, 2011, pp. 318-325, http://dx.doi.org/10.1109/mascots.2011.41.

[13] Y. Kim, A. Gupta, B. Urgaonkar, P. Berman, and A. Sivasubramaniam, "Hybridstore: A cost-efficient, high-performance storage system combining ssds and hdds," in Modeling, Analysis \& Simulation of Computer and Telecommunication Systems (MASCOTS), 2011 IEEE 19th International Symposium on. IEEE, 2011, pp. 227-236, http: //dx.doi.org/10.1109/mascots.2011.64.

[14] N. Zhang, J. Tatemura, J. M. Patel, and H. Hacigümüş, "Towards costeffective storage provisioning for dbmss," Proceedings of the VLDB Endowment, vol. 5, no. 4, pp. 274-285, 2011, http://dx.doi.org/10.14778/ 2095686.2095687.

[15] H. Ouarnoughi, J. Boukhobza, F. Singhoff, and S. Rubini, "A cost model for virtual machine storage in cloud iaas context," in Parallel, Distributed, and Network-Based Processing (PDP), 2016 24th Euromicro International Conference on. IEEE, 2016, pp. 664-671, http://dx.doi.org/10.1109/pdp.2016.119.

[16] D. Boukhelef, J. Boukhobza, and K. Boukhalfa, "A cost model for dbaas storage," in International Conference on Database and Expert Systems Applications. Springer, 2016, pp. 223-239, http://dx.doi.org/10.1007/ 978-3-319-44403-1_14.

[17] M. Hadji and D. Zeghlache, "Mathematical programming approach for revenue maximization in cloud federations," IEEE transactions on cloud computing, vol. 5, no. 1, pp. 99-111, 2017, http://dx.doi.org/10.1109/tcc. 2015.2402674

[18] Z. Wen, J. Cała, P. Watson, and A. Romanovsky, "Cost effective, reliable and secure workflow deployment over federated clouds," IEEE Transactions on Services Computing, vol. 10, no. 6, pp. 929-941, 2017, http://dx.doi.org/10.1109/tsc.2016.2543719.

[19] L. Zhang, C. Wu, Z. Li, C. Guo, M. Chen, and F. C. Lau, "Moving big data to the cloud: An online cost-minimizing approach," IEEE Journal on Selected Areas in Communications, vol. 31, no. 12, pp. 2710-2721, 2013, http://dx.doi.org/10.1109/jsac.2013.131211.

[20] W. Xiao, W. Bao, X. Zhu, and L. Liu, "Cost-aware big data processing across geo-distributed datacenters," IEEE Transactions on Parallel and Distributed Systems, vol. 28, no. 11, pp. 3114-3127, 2017, http://dx.doi. org/10.1109/tpds.2017.2708120.

[21] Y. Mansouri, A. N. Toosi, and R. Buyya, "Cost optimization for 
dynamic replication and migration of data in cloud data centers," IEEE Transactions on Cloud Computing, 2017, http://dx.doi.org/10.1109/tcc. 2017.2659728.

[22] A. Khosravi, L. L. Andrew, and R. Buyya, "Dynamic vm placement method for minimizing energy and carbon cost in geographically distributed cloud data centers," IEEE Transactions on Sustainable Computing, vol. 2, no. 2, pp. 183-196, 2017, http://dx.doi.org/10.1109/tsusc. 2017.2709980.

[23] K. Le, R. Bianchini, J. Zhang, Y. Jaluria, J. Meng, and T. D. Nguyen, "Reducing electricity cost through virtual machine placement in high performance computing clouds," in Proceedings of 2011 International Conference for High Performance Computing, Networking, Storage and Analysis. ACM, 2011, p. 22, http://dx.doi.org/10.1145/2063384. 2063413.

[24] H. Yuan, J. Bi, W. Tan, and B. H. Li, "Cawsac: Cost-aware workload scheduling and admission control for distributed cloud data centers," IEEE Transactions on Automation Science and Engineering, vol. 13, no. 2, pp. 976-985, 2016, http://dx.doi.org/10.1109/tase.2015.2427234.

[25] S. Rebai, M. Hadji, and D. Zeghlache, "Improving profit through cloud federation," in Consumer Communications and Networking Conference (CCNC), 2015 12th Annual IEEE. IEEE, 2015, pp. 732-739, http: //dx.doi.org/10.1109/cenc.2015.7158069.

[26] M. Hadji, B. Aupetit, and D. Zeghlache, "Cost-efficient algorithms for critical resource allocation in cloud federations," in Cloud Networking (Cloudnet), 2016 5th IEEE International Conference on. IEEE, 2016, pp. 1-6, http://dx.doi.org/10.1109/cloudnet.2016.11.

[27] J. Boukhobza, "Flashing in the cloud: Shedding some light on nand flash memory storage systems," in Data Intensive Storage Services for Cloud Environments. IGI Global, 2013, pp. 241-266, http://dx.doi.org/ 10.4018/978-1-4666-3934-8.ch015.

[28] J. Boukhobza and P. Olivier, Flash Memory Integration: Performance and Energy Issues. Elsevier, 2017, https://www.elsevier.com/books/ flash-memory-integration/boukhobza/978-1-78548-124-6.

[29] D. Lee, C. Min, and Y. I. Eom, "Effective flash-based ssd caching for high performance home cloud server," IEEE Transactions on Consumer Electronics, vol. 61, no. 2, pp. 215-221, 2015, http://dx.doi.org/10.1109/ tce.2015.7150596.

[30] C. Wu and R. Buyya, Cloud Data Centers and Cost Modeling: A complete guide to planning, designing and building a cloud data center. Morgan Kaufmann, 2015, https://www.elsevier.com/books/ cloud-data-centers-and-cost-modeling/wu/978-0-12-801413-4.

[31] J. Tai, B. Sheng, Y. Yao, and N. Mi, "Live data migration for reducing sla violations in multi-tiered storage systems," in Cloud Engineering (IC2E), 2014 IEEE International Conference on. IEEE, 2014, pp. 361-366, http://dx.doi.org/10.1109/ic2e.2014.8.

[32] W. Xiao, X. Lei, R. Li, N. Park, and D. J. Lilja, "Pass: a hybrid storage system for performance-synchronization tradeoffs using ssds," in Parallel and Distributed Processing with Applications (ISPA), 2012 IEEE 10th International Symposium on. IEEE, 2012, pp. 403-410, http://dx.doi.org/10.1109/ispa.2012.59.

[33] Square kilometre array. https://www.skatelescope.org/.

[34] A. Simonet, A. Lebre, and A.-C. Orgerie, "Deploying distributed cloud infrastructures: Who and at what cost?" in Cloud Engineering Workshop (IC2EW), 2016 IEEE International Conference on. IEEE, 2016, pp. 178-183, http://dx.doi.org/10.1109/ic2ew.2016.48.

[35] N. Grozev and R. Buyya, "Inter-cloud architectures and application brokering: taxonomy and survey," Software: Practice and Experience, vol. 44, no. 3, pp. 369-390, 2014, http://dx.doi.org/10.1002/spe.2168.

[36] A. N. Toosi, R. N. Calheiros, and R. Buyya, "Interconnected cloud computing environments: Challenges, taxonomy, and survey," $A C M$ Computing Surveys (CSUR), vol. 47, no. 1, p. 7, 2014, http://dx.doi. org/10.1145/2593512.

[37] N. K. Gill and S. Singh, "A dynamic, cost-aware, optimized data replication strategy for heterogeneous cloud data centers," Future Generation Computer Systems, vol. 65, pp. 10-32, 2016, http://dx.doi.org/10.1016/ j.future.2016.05.016.

[38] R. N. Calheiros, R. Ranjan, A. Beloglazov, C. A. De Rose, and R. Buyya, "Cloudsim: a toolkit for modeling and simulation of cloud computing environments and evaluation of resource provisioning algorithms," Software: Practice and experience, vol. 41, no. 1, pp. 23-50, 2011, http://dx.doi.org/10.1002/spe.995.
APPENDIX A: NOTATION TABLE

\begin{tabular}{|c|c|}
\hline Symbol & Meaning \\
\hline \multicolumn{2}{|l|}{ Federation } \\
\hline$F$ & Federation of a set of CPs \\
\hline$C P^{d}$ & cloud provider $d$ \\
\hline $\begin{array}{l}\text { Cap } \text { max }_{\text {res }}, \\
\text { Cap }_{\text {idl }_{\text {res }}, p_{r s c}}\end{array}$ & $\begin{array}{l}\text { The total and the idle capacities of storage resource res } \\
\text { and its price. }\end{array}$ \\
\hline$F_{\text {res }}^{d}$ & The insourcing price of storage resource res of $C P^{d}$ \\
\hline \multicolumn{2}{|l|}{ Customers } \\
\hline$U_{i n t}, u_{k}$ & The set of internal customers and $k t h$ customer \\
\hline$U_{e x t}, u_{k^{\prime}}^{d^{\prime}}$ & $\begin{array}{l}\text { The set of external customers and } k^{\prime} \text { th external } \\
\text { customer of } C P^{d} \text { (internal customer of the cloud } C P^{d^{\prime}} \text { ) }\end{array}$ \\
\hline$w l_{k}$ & the workload of $u_{k}$ \\
\hline$i o p s_{s l a, k}, l t c_{s l a, k}$ & The requested performance in terms of IOPS and latency \\
\hline $\begin{array}{l}p n_{k}, p n_{\text {iops }, k} \\
p n_{l t c, k}\end{array}$ & $\begin{array}{l}\text { The penalty function, and its parts iops penalty } \\
\text { and latency penalty }\end{array}$ \\
\hline iops $_{\text {offered }}\left(u_{k}\right)$ & The IOPS offered to customer $u_{k}$ \\
\hline ltc $c_{\text {offered }}\left(u_{k}\right)$ & The latency offered to customer $u_{k}$ \\
\hline \multicolumn{2}{|l|}{ Storage } \\
\hline$S C, s c_{j}$ & The set of storage classes, The $j t h$ storage class \\
\hline$s c_{j, i n t}, s c_{j, e x t}$ & The internal and external customers storage parts \\
\hline$c_{s c_{j}}, p_{s c_{j}}, w o_{s c_{j}}$ & The capacity, price and wear out of $s c_{j}$ \\
\hline \multicolumn{2}{|l|}{ Bandwidth } \\
\hline$b w, p_{b w}$ & The bandwidth and its purchased cost \\
\hline$b w_{i n t}, b w_{e x t}$ & The internal and external customers bandwidths \\
\hline \multicolumn{2}{|l|}{ Objects } \\
\hline$o_{i, k}, s_{o_{i, k}}$ & The $i t h$ object of customer $u_{k}$ and its size \\
\hline$r e q_{o p, o_{i, k}}$ & The average IOPS of type $o p$ issued to the object $o_{i, k}$ \\
\hline$O_{m, i n t}, O_{m, e x t}$ & $\begin{array}{l}\text { The set of internal (external, respectively) customers } \\
\text { objects to migrate between internal storage classes }\end{array}$ \\
\hline$A[i, j], B[i, j]$ & $\begin{array}{l}\text { the internal and external customers objects } \\
\text { placement matrices }\end{array}$ \\
\hline \multicolumn{2}{|l|}{ General } \\
\hline$T$ & Period of time \\
\hline$T_{s p}$ & The internet subscription period \\
\hline \multicolumn{2}{|l|}{ Costs } \\
\hline Cost $_{p l c, T}$ & The total placement cost \\
\hline Cost $_{p l c_{\text {int }}, T}$ & The internal customers objects placement cost \\
\hline Cost $_{p l c_{e x t}, T}$ & The external customers objects placement cost \\
\hline Cost $_{\text {lcl }}$ int,$T$ & The local placement costs of the internal customers \\
\hline Cost $_{l c l_{e x t}, T}$ & The local placement costs of the external customers \\
\hline Cost $_{\text {pnt }}$ int,$T$ & The penalty costs of the internal customers \\
\hline Cost $_{p n t_{e x t}, T}$ & The penalty costs of the external customers \\
\hline Cost $_{\text {pnt }} t_{i o p s, T}$ & IOPS penalty costs \\
\hline Cost $_{\text {pnt }} t_{\text {ltc }}, T$ & Latency penalty costs \\
\hline Cost $_{\text {stgint }_{\text {in }}, T}$ & The storage cost of the internal customers objects \\
\hline Cost $_{\text {stg }_{\text {ext }}, T}$ & The storage cost of the external customers objects \\
\hline Cost $_{m g r_{i n t}, T}$ & The inner migration costs of the internal customers objects \\
\hline Cost $_{m g r_{e x t}, T}$ & The inner migration costs of the external customers objects \\
\hline Cost $_{\text {out }}$ src $_{\text {c }}, T$ & The outsourcing cost \\
\hline Cost $_{i n_{s r c}, T}$ & The insourcing cost \\
\hline Cost $_{\text {geo }}{ }_{m g r}, T$ & The geo-migration cost \\
\hline Cost $_{b c k_{m i g}, T}$ & ,The back-migration cost \\
\hline Cost $_{r e_{m g r}, T}$ & The re-migration cost \\
\hline Cost $_{g e o_{r p l}, T}$ & The geo-replication cost \\
\hline Cost $_{a d d_{r e p}, T}$ & The cost of adding a replica \\
\hline Cost $_{\text {syn }_{\text {rep }}, T}$ & The cost of synchronizing replicas \\
\hline Cost $_{r d, T}$ & The local read cost \\
\hline Cost $_{r d_{\text {erg }}, T}$ & The read energy cost \\
\hline Cost $_{r d_{e d r}, T}$ & The read wear out cost \\
\hline $\operatorname{Cost}_{w r, T}$ & The local write cost \\
\hline Cost $_{w r_{\text {erg }}, T}$ & The write energy cost \\
\hline Cost $_{w r_{e d r}, T}$ & The write wear out cost \\
\hline Cost $_{b w, T}$ & The local consumed internet bandwidth cost \\
\hline Cost $_{b w_{a m z}, 1}$ & The amortized internet bandwidth over one unit of time \\
\hline Cost $_{n t w_{\text {out }}, T}$ & The external outgoing network cost \\
\hline Cost $_{e x t_{o c c}, T}$ & The external occupation cost \\
\hline Cost $_{\text {ext }} w l d, T$ & The external workload cost \\
\hline Cost $_{e x t_{p l t}, T}$ & The penalty cost \\
\hline
\end{tabular}

